\title{
Impact of Ground Level Enhancement (GLE) Solar Events on Soft Error Rate for avionics
}

\author{
G. Hubert, S. Aubry, J.A. Clemente
}

\begin{abstract}
This work proposes to study the impact of GLE induced by Extreme Solar Flares on the SER for flights representative to the world-air traffic. A GLE physical model was confronted to cosmic ray variations measured with neutron monitors during the GLEs 5 and 69. Based on said GLE physical model and SEU occurrence models, investigations were performed to assess the SER during these GLEs for a bulk 90-nm SRAM COTS SRAM. The main conclusion is that the GLE impact must be considered in the risk assessment, which requires a physical description of the additional solar cosmic rays and a realistic consideration of the air traffic.
\end{abstract}

\section{INTRODUCTION}

$\mathrm{T}$ HE Earth is constantly bombarded by cosmic rays (CRs) that can be of either galactic or solar origin. When galactic cosmic rays (GCRs) strike the atmosphere, they can interact with the nuclei of oxygen and nitrogen molecules to generate a cascade of secondary particles including neutrons, protons and electrons [1][2]. The secondary radiation builds up to a maximum at around $18 \mathrm{~km}$ and then attenuates down to sea level. The fluxes of particles at subsonic flight levels (12 km) are about 300 times greater than at sea level whereas at $18 \mathrm{~km}$, they are about 500 times more intense. Therefore, electronic systems are exposed to secondary CRs at altitude aircraft [3]-[6]. On the other hand, the geomagnetic field provides greater shielding at the equator than at the poles and the secondary radiation increases by about a factor of five between the equator and latitudes of around 60 degrees beyond which the levels flatten off with increasing latitude.

Single Event Effects (SEEs) are individual events which occur when a single incident particle deposits enough energy to cause an effect in a device. Among the many variations of effects, the most studied ones are the so-called Single-Event Upsets (SEUs), corresponding to the change in state of one or several storage elements inside a device or a system. More precisely, a single storage location upset provoked by one particle is named Single Bit Upset (SBU), whereas an upset in multiple storage locations is named Multiple Cell Upset (MCU). The Soft Error Rate (SER) is used to express the rate at which a device or a system is disturbed or it is predicted to encounter a SEE. It is typically expressed in number of failures per time unit and it can be

Guillaume Hubert is with the ONERA/DPHY, University of Toulouse, France. He is with the Department of Physics, Instrumentation, Environment and Space (e-mail: guillaume.hubert@onera.fr).

Sébastien Aubry is with the ONERA/DTIS, University of Toulouse, France (e-mail: sebastien.aubry@onera.fr).

Juan Antonio Clemente is with the computer Architecture Department, Facultad de Informática, Universidad Complutense de Madrid (UCM), Spain (e-mail: ja.clemente@fdi.ucm.es). 
converted in number of Failures-In-Time (FIT) or Mean Time Between Failures (MTBF). Thus, in order to evaluate the SER, methods need to combine radiation field, the SEE assessment and eventually consider the aircraft's flight path.

The Sun can influence the neutron [7][8] flux within the atmosphere in two main ways: 1) the impact of solar activity on the intensity of GCRs reaching the Earth, and 2) occasional solar flares resulting in large bursts of solar energetic particles arriving in the atmosphere which create a brief increase in the atmospheric neutron flux. Classically, the GCR spectra near the Earth are parameterized using the Force-Field model [9] with an only time-dependent parameter, which is the modulation solar potential $\varphi$.

Solar Energetic Particles (SEPs) are high-energy particles coming from the Sun which had been first observed in the early 1940s. SEPs can originate from two processes: energization at a solar flare site or by shock waves associated with Coronal Mass Ejections (CMEs). SEPs can produce additional instantaneous atmospheric effects during relatively short periods [10][11]. SEPs are typically characterized by low-energy-particles (i.e. $<100 \mathrm{MeV}$ for protons), whose effects are limited to the upper atmosphere. In this case, the analytical approximation of direct ionization [2] can be applied. More rarely, solar proton spectra can extend up to 1-10 GeV, energies that are high enough to induce cascades of particles in the atmosphere. In this case, Ground level enhancements (GLEs) are observed and they can reinforce the atmospheric air shower cascade of secondary particles. Thus, GLEs correspond to increases in the CR intensity recorded by ground-based detectors named Neutron Monitors (NMs). GLEs are invariably associated with large solar flares but the acceleration mechanism producing particles with energies of up to tens of $\mathrm{GeV}$ is not well understood yet. What is certain is that GLEs can produce large local differences in the secondary particle spectra, up to two orders of magnitude. These events are typically not considered in the SER assessment for terrestrial and avionic fields, probably because it requires a fine description of the physical mechanisms.

Significant works [12]-[14] referring to SEEs in avionics during quiet solar conditions were presented since the 1990s, and unfortunately not for critical solar flares. However, solar CR increases induced by solar flares lasting until 12 hours in highenergy are of concern because they impact the probability of occurrence of SEEs in aircraft systems. Analyses of events recorded in the past have shown that the solar flare impact concerns primarily high latitudes, but it can be extended in some cases to low latitudes, which means that a significant proportion of commercial flight routes could be affected. From a practical point of view, SEPs are characterized by a wide spectrum of energies, which is not possible to predict in an accurate way. Moreover, the first particles impact the high-atmosphere within a few minutes of seeing the associated solar flare. Consequently, neither a practical assessment of an event that would occur, nor its associated SEE impact can be provided.

To mitigate three hazards associated to the GLEs, avionic systems routinely incorporate techniques based on redundancy and majority voting. Notwithstanding these design approaches, additional specific engineering steps could be required to minimize the risk from SEPs. The IEC standard on avionics atmospheric radiation [15]-[17] also provides some methodologies to reach compliance of avionics that will operated in an atmospheric radiation environment, including solar events. However, more 
research is still needed to understand these mechanisms in detail. For instance, the so-called "Carrington event", occurred in 1859, was not recorded with radiation detectors but characterized by aurora borealis observed until tropical latitudes. The Carrington event is often considered as reference and it is systematically mentioned in standard. Nevertheless, analyses [18] based on ${ }^{10} \mathrm{Be}$ concentration in ice cores do not support the hypothesis of extremely abundant SEPs related to the Carrington flare. This reflects some uncertainty in the apprehension of the GLEs, therefore it seems appropriate to propose systematic investigations based on physical models.

The objective of this work is to study the GLE impact on the SER for typical avionic flights. The GLE model includes anisotropic and spectral solar CR descriptions, and the atmospheric secondary spectra can be simulated using air-shower modelling. Elements of validation are presented by comparisons of CR calculations and measurements issued from NMs. Indeed, NMs record most of the time the Galactic Cosmic Rays (GCRs) and occasionally Solar Cosmic Rays (SCRs) emitted by the Sun during strong solar events. They are termed "ground level enhancements" (GLE) when solar particles have sufficient energy and intensity to raise radiation levels on Earth's surface to the degree that they are readily detected by NMs. Then, SER assessments during a quiet solar activity and GLE events are investigated considering realistic flight paths. Two approaches were proposed. The first one focuses on the impact of the delay between the GLE occurrence and the flight departure. The second approach reaches to consider the impact of a fixed GLE universal time (UT) with respect to the global air traffic.

\section{MODELING PLATFORMS AND VALIDATIONS}

\section{A. Simulation of the Extensive Air Showers}

The Force-Field approximation model [9] is typically used to describe the GCR spectra. This model provides a simple parametric approximation of the GCR spectrum and it depends only on the modulation potential $\varphi$. Nevertheless, actual SCR spectra observed on Earth depends on some characteristics, namely: the source site, the acceleration mechanism, the coronal transport, the ejection profile as well as the transport of accelerated particles through the interplanetary magnetic field. During a critical GLE, the solar proton spectra can extend up to 1-10 GeV, energies that are high enough to induce cascades of particles in the atmosphere. CR variations may increase by a factor that may range from small percentage to more than 10 in a short period of time, depending on the measurement location and on the energy of the arriving particles. To model these events, it is necessary to consider the anisotropy and the spectral dynamics.

An anisotropy function represents the distribution of SCR particles at the top of the atmosphere during the GLE, which can be studied from data analysis from several CR detectors, such as NMs. In previous works [19], a modelling approach applied to the GLE 69 event was presented. According to that model, the SCR can be expressed by means of the following equation: 
Where $\Psi(\Omega, R, t)$ is the anisotropy function reflecting the angular dependence of the flux for particles with rigidity $R$ coming from the asymptotic direction $\Omega$, and $S(E, t)$ is the differential SCR rigidity spectrum in a solid angle of asymptotic direction. The differential SCR rigidity spectrum can be expressed as:

$J(E, t)=J_{0}(E, t) \cdot R(E)^{-\gamma(t)}$

Where $J_{0}(E, t)$ and $\gamma(t)$ are the SEP intensity and the power index, respectively. To determine the secondary spectra, an approach named ATMORAD [20] describing the atmospheric radiation was presented in a previous work [20]. It was built using GEANT4 [32][33] simulations of extensive air showers, and it allows to determine fluxes of secondary particles at various altitudes induced by showers and taking into account the magnetic field impact. Comparisons with standard approaches and measurements were presented in [20]-[23].

From the point of view of the assessment of the effects, the dynamic GLE can be described according to two approaches: a delay between the flight departure and the occurrence of an event applied to all the flights, or a chosen universal time applied to all the flights.

\section{B. Validations of calculated CR variations during a GLE}

The first step for the SER assessment during GLE events presented in this work consisted in validating the SCR model above described taking into account the anisotropic properties of the solar proton spectrum. Thus, two large intensity GLEs were considered. Firstly, the event occurring on 20 January 2005 (GLE 69) as the most recent large-intensity GLE with the advantage of having being monitored on a large number of NMs [24]-[26]. Secondly, the GLE which occurred on 23 February 1956 (GLE 5) [26] was investigated because it constitutes a reference as the most intense proton event observed since 1942.

Fig. 1 and Fig. 2 present a synthesis of the comparison between measurements and calculations of CR variation for GLE 69 (Fig. 1) and GLE 5 (Fig. 2), respectively. Data were recorded in several NM stations distributed in very different locations. Concerning the GLE 69, the South Pole, McMurdo (in the Southern Hemisphere), Thule and Tibet stations (in the Northern hemisphere) were considered. Calculations are relevant except for Tibet station (overestimation of factor 5). It is important to note the anisotropy of the GLE effect between both hemispheres. Indeed, GLE 69 is known to have particularly impacted the southern hemisphere and its polar zone. 

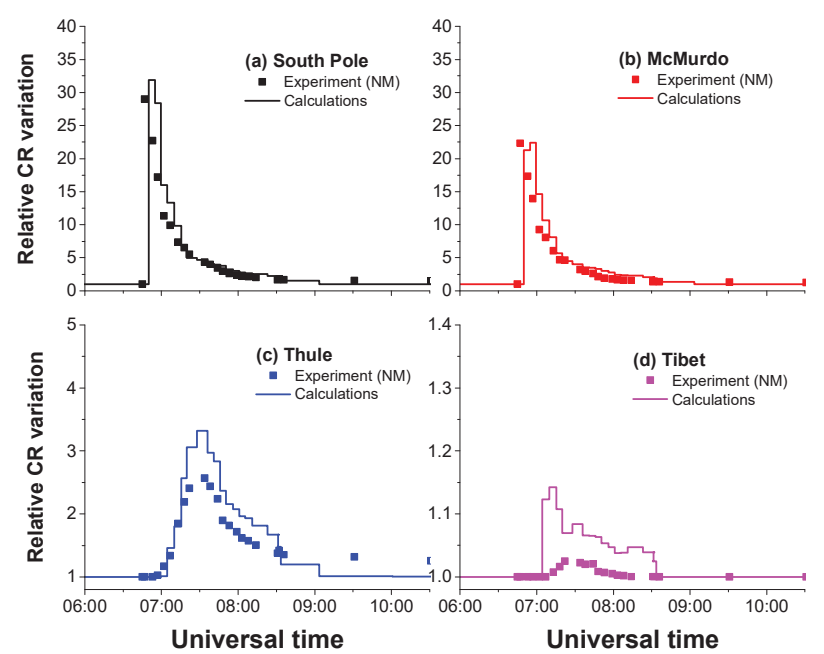

Fig. 1: Cosmic ray variations measured and calculated during the GLE 69 in the South Pole (a), McMurdo (b), Thule (c) and Tibet stations (d).
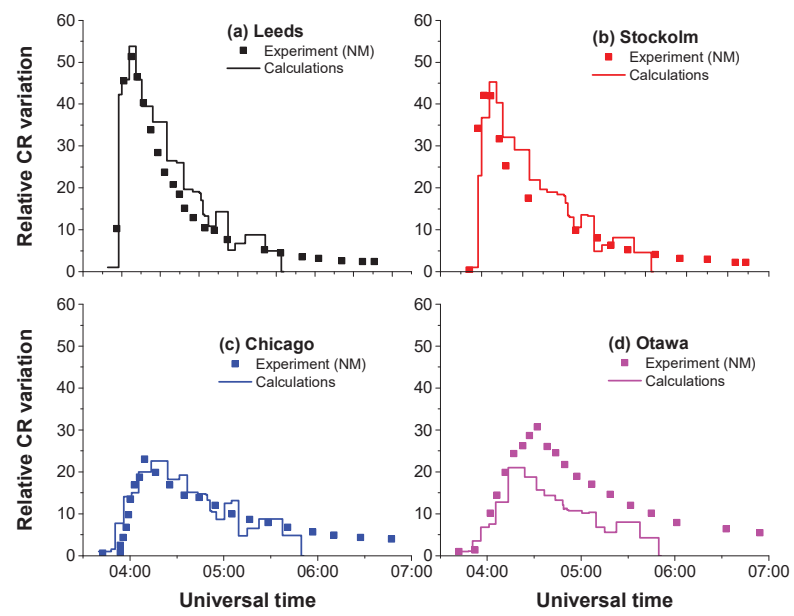

Fig. 2: Cosmic ray variations measured and calculated during the GLE 5 in Leeds (a), Stockholm (b), Chicago (c) and Ottawa (d).

The GLE 5 was recorded by about ten NMs [24][25] located over a wide range of longitudes and rigidities. The CR variation amplitudes were respectively 50, 45, 35 and 25 for Leeds, Stockholm, Ottawa and Chicago (Fig. 1(a) and Fig. 1(b)). The SCR spectra and the anisotropy function were described by Belov et al. [19], allowing to parametrize the GLE model for each NM station and to compare the CR variations issued from data analyses and calculations. Orders of magnitude are acceptable, despite systematic overestimations by a factor of 20-30 \% for Leeds, Stockholm and Chicago. Despite its proximity to Chicago, results 
obtained for the Ottawa station are underestimated in the order of $40 \%$. Moreover, dynamics are quite correct for the three stations, whereas there is a significant difference observed for Ottawa.

There are few data on CR effects during GLEs and they relate exclusively the dose assessment for aircrews. For a Paris Washington D.C. flight, the evaluated dose value during the GLE 5 is around $1.75 \mathrm{mSv}$ [27][28], whereas the typical dose during quiet solar activity is around $50 \mu \mathrm{Sv}$. Calculations based on the GLE model and ATMORAD give a dose value of $1.6 \mathrm{mSv}$. An important remark is that the risk for a typical transatlantic flight seems to be 30 times higher during the GLE 5 than during a quiet solar activity. These data are interesting because, although the ambient dose and SEE effects are quite different, the energy range is similar and it may be presumed that the GLE impact on SER should be significant. This is particularly true for flights whose duration is of the same order of magnitude as the disturbances induced by a GLE.

\section{SEE modeling and validation}

1) SEE modelling based on multi-physics approach

SEE risks for a given device can be deduced from experimental tests or by using modelling. During the last 20 years, multiscales modelling, physics-based Monte-Carlo (MC) or analytical simulations have been widely developed. The MUSCA SEP3 [29][30] methodology was developed by the authors for sequentially modelling all physical mechanisms involved in the SEE occurrence, from the system down to the semiconductor target. This approach allows to consider the device, as well as its local and global environment (including shielding, package, etc.). The device description includes the active zones (topology, layout and dimensions), passivation and metallization layers. The MC approach consists in transporting the particle within the device and the primary or secondary ion energy loss along the path is simulated by using the stopping powers pre-calculated with the SRIM code [31]. The simulation of nuclear reactions induced by primary protons or neutrons is performed by inputting precalculated (using GEANT4 [32][33]) nuclear reactions for various incident energies and material. Finally, primary and secondary ions are responsible for production of electron-hole pairs in the device. The next step is to simulate the transport and the collection mechanisms inducing charge level or transient pulse in sensitive nodes. Physical models were developed using Technology Computer-Aided Design (TCAD) simulations (i.e. a functional simulation of the device considering Poisson and continuity equations). These kind of simulations are commonly used to develop and optimize semiconductor processing technologies and devices. The impact of the particle can be deduced by associating charges/transients with criteria. The SER calculation involves associating the radiation field spectrum, its associated SEE cross section, the angular properties and the material environment. 
SEE modelling allows to determine a set of cross sections for a given particle $k$ (neutron, proton, muon), a given location and for several energies. From the cross section and the secondary spectral fluence rate issued from ATMORAD, it is possible to calculate $S E R_{k}(t)$, the soft error rate relative to a given location and the particle $k$ :

$S E R_{k}(t)=\int_{\text {spectrum }} \sigma_{S E E, k}(E) \cdot \frac{\delta \varphi_{k}\left(E, h, R_{C}, t\right)}{\delta E} \cdot \delta E$

$\sigma_{S E E, k}(E)$ is the SEE cross section defined as the ratio between the event number and the total fluence (i.e. particle number per surface unit). The term $\delta \varphi_{k}\left(E, h, R_{C}, t\right) / \delta E$ corresponds to the secondary spectral fluence rate of particle $k$ with the energy $E$ at altitude $h$ and considering a cutoff rigidity of $R_{c}$ (which depends on the geomagnetic latitude). The secondary spectral fluence rate is expressed in particle per $\mathrm{cm}^{2}$ per $\mathrm{s}$ and per MeV. The global SER relative to a flight, named SER flight, is obtained by combining all particle contributions and by integration over the time flight. For each time flight value, the altitude, the longitude and the latitude (defining the cutoff rigidity) were implicitly considered.

$S E R_{\text {flight }}=\sum_{k=n, p, \mu} \int_{t(p a t h)} S E R_{k}(t) \cdot \delta t$

\section{2) Previous works and analyses}

Various Commercial-Off-The-Shelf (COTS) SRAMs were exposed to neutron and proton facilities to investigate SEE sensitivities including SBU/MCU analyses and studying the impact of voltage scaling. The CY62167EV30LL-45ZXI CMOS SRAM manufactured by Cypress was particularly studied in the framework of ground and in-flight tests. The facility/experiment details and analyses were presented in previous works and summarized in Table I. Thus, the device was tested with a thermal neutron beam at Institute Laue-Langevin (ILL), with a $14.2 \mathrm{MeV}$ neutron beam at the GENEPI2 facility and with a $450 \mathrm{MeV}$ neutron facility at Triumf. For proton fields, energies from 50 to $180 \mathrm{MeV}$ were considered at the Kernfysisch Versneller Instituut (KVI). From 2007 to 2012, an experimental platform including 1 Gb memory built from this device was used for SEU detection during commercial flights, stratospheric balloon flights and in high-altitude stations. Data analyses included SBU/MCU sensitivities and/or ultralow bias voltage assessment. Experimental data were used to validate the SEU occurrence model to consider operational cases. Details of technological analyses were presented in [4], i.e. the elementary SRAM cell topology including transistor locations and surfaces. The cell area is around $1.02 \mu \mathrm{m}^{2}(1.2 \mu \mathrm{m} \times 0.85 \mu \mathrm{m})$ and drain surfaces have been estimated to be around $0.2 \mu \mathrm{m} \times 0.2 \mu \mathrm{m}$. 


\begin{tabular}{lll}
\hline Ref. & Facility/experiment details & Descriptions \\
\hline$[34][35]$ & Commercial flights & 1Gbit platform, SER \\
\hline$[36]$ & ASP, 14.2 MeV neutron & SBU and MCU \\
\hline & Triumf, 450 MeV neutrons & \\
{$[37]$} & Themal neutron (ILL) & \\
& KVI, $50,100,180 \mathrm{MeV}$ protons & SBU and MCU \\
\hline$[38][39][40]$ & Balloon flight & \\
{$[30]$} & GENEPI2, 14.2 MeV neutrons & SBU and MCU, ultralow bias voltage \\
& High altitude stations & SER: Pic du Midi (France), Puno (Peru) \\
\hline
\end{tabular}

All results and analyses presented in previous works [30]-[40] have shown the relevance of MUSCA SEP3 simulations to predict the SBU/MCU SER and bias voltage impact on the device sensitivities. In addition, preliminarily results presented in [34][35] allowed to assess in-flight SER with satisfactory relevance, despite a simplified radiation field and flight path modelling. All these elements guarantee that the technological and occurrence models are particularly relevant for this technology and that they can be used to investigate the impact of the radiative environment. Certainly a more integrated technological node would have matched more modern aircraft avionics; this does not alter the purpose of studying the impact of GLEs on the SER.

\section{3) In-flight data}

As presented in [21]-[23], realistic flight paths can be extracted from the Eurocontrol Demand Data Repository (DDR) [41], including some descriptions from flight plan, integrating regulation or updated with radar data from CFMU (Central Flow Management Unit). Databases were generated by using DDR, containing more than 100.000 flight paths, which makes it possible to have a representative sample of the commercial air traffic. Each path was described by means of the following data: date, universal time, longitude, latitude and altitude.

A large number of paths were extracted from DDR databases for flights, for which the experimental platform was embedded, i.e. Los Angeles - Paris, Buenos Aires - Paris and Madrid - Buenos Aires flights [34]. Thus, Fig. 3 presents the normalized probability of occurrence of different numbers of SEUs estimated for the Los Angeles - Paris, Buenos Aires - Paris and Madrid - Buenos Aires flights. Several hundreds of paths are considered for each flight, inducing a significant variability in latitudes and altitudes. Path variabilities imply using a distribution to translate the SEU risk, and not an average value which refers to a single path. The measured SER was referenced and shows a good concordance with calculations. It is interesting to note the importance of the flight paths in the SER level. For instance, for the Los Angeles - Paris flight, the minimum, average and maximum SEU numbers are equal to $7.5,14.8$ and 20 , respectively. These differences are due to variations in altitude and latitude, which illustrates the importance of considering the path description. 


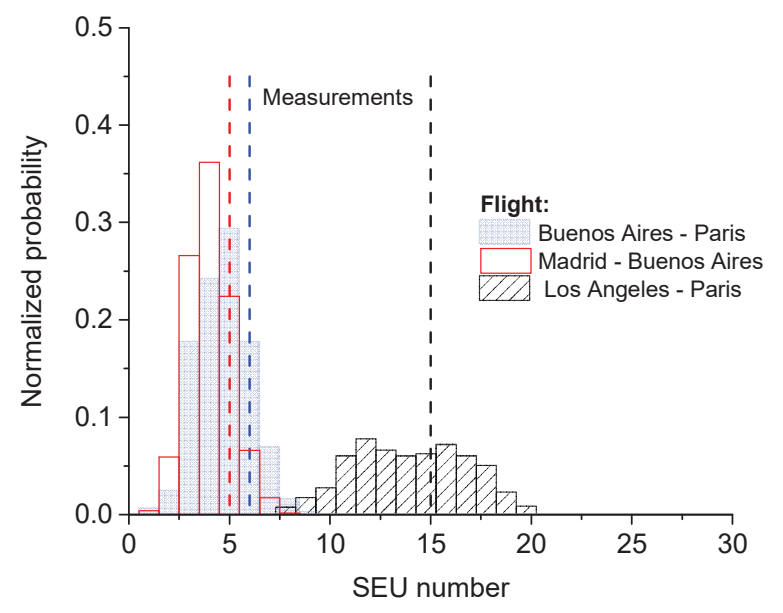

Fig. 3: Distribution of the SEU number calculated for the Los Angeles - Paris, Buenos Aires - Paris and Madrid - Buenos Aires flights. The measured SEU numbers recorded during flights were added.

\section{IMPACT OF GLES ON THE SER FOR COMMERCIAL FLIGHTS}

\section{A. Statistical analyses of the SER during quiet conditions}

The objective is to assess the SER during a quiet solar period (i.e. solar modulation of $700 \mathrm{MV}$ ) in considering flight paths described in the European air traffic database. Four flights were selected and, in order to increase statistics, a high number of paths $(\sim 500)$ were selected for each one of them. Thus, flights linking Paris with Los Angeles, New York, Tokyo and Sao Paulo are considered and indeed, they are representative to the continents or sub-continents of European international air traffic. There is a great diversity of trajectories for a given flight. It is especially true for Paris - Los Angeles, Paris - New York and Paris Tokyo. Path characteristics necessarily impact SEU risks, in quiet conditions or during a solar event for which recent works had showed the importance of path characteristics in the dose assessment [22].

Some anomalies can be also identifiable depending on the trajectory, whose mere existence reflects flight plan modifications, probably due to weather conditions. Fig. 4 presents the normalized SER probability, expressed in SEU number per flight and Gbit, for (a) Paris - Los Angeles, (b) Paris - New York, (c) Paris - Tokyo and (d) Paris - Sao Paulo routes. The flight direction (from or to Paris) is considered to be interesting because the flight durations are not the same due to the prevailing atmospheric currents (East - West), and the air corridors. Analyses focusing on Paris - New York flights showed that SER ranges from 5 to $13 \mathrm{SEU} / \mathrm{Gb}$ for the East-West (EW) direction, while it ranges from 7 to $22 \mathrm{SEU} / \mathrm{Gb}$ for the West-Est (WE) direction. The difference is clearly significant and it can be observed for other flights. The average, the maximum and the minimum SER values for each selected flights were added in Fig. 3. 

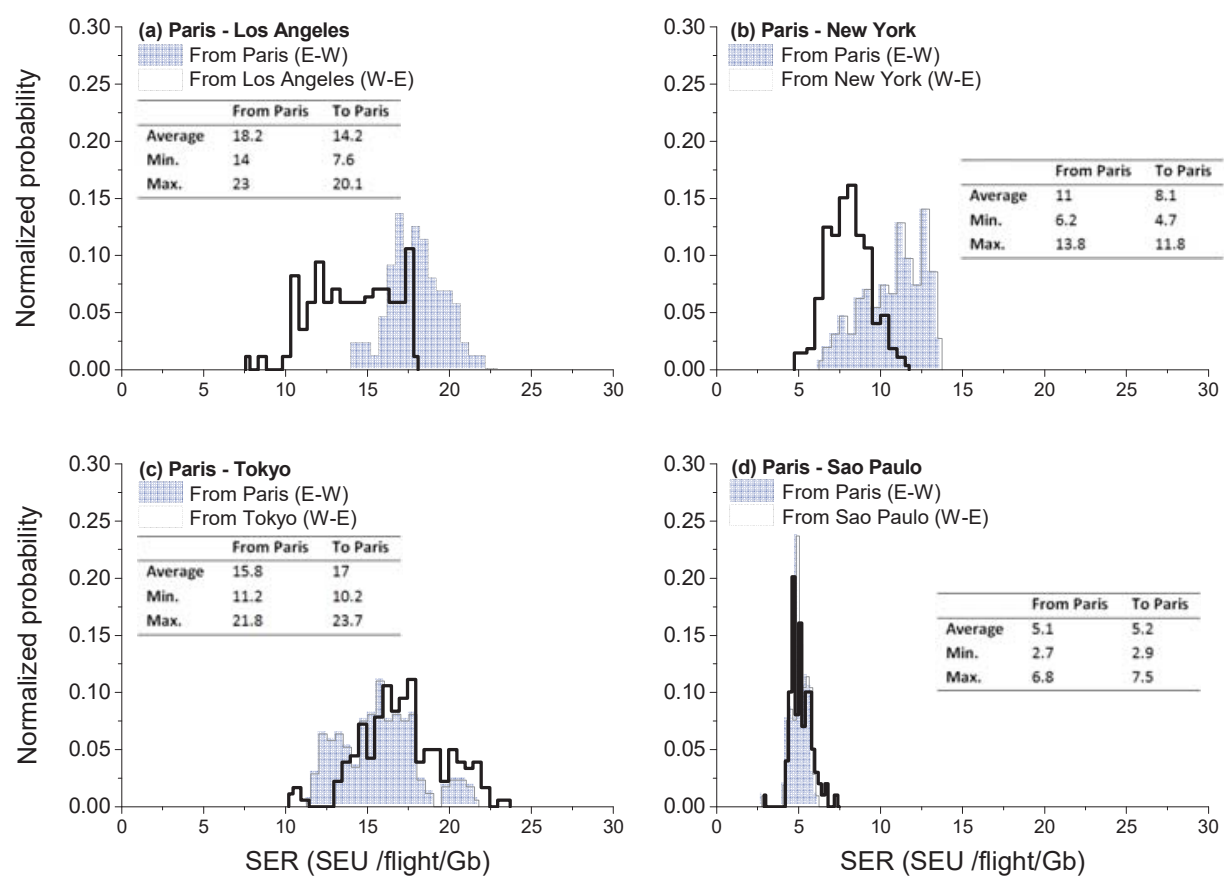

Fig. 4: Normalized probability of SER for (a) Paris- Los Angeles, (b) Paris- New York, (c) Paris - Tokyo and (d) Paris - Sao Paulo routes. Results are presented in distinguishing the flight direction (from / to Paris). Average, minimum and maximum SER were added.

\section{B. Statistical analyses of the SER considering a GLE scenario}

These analyses concern the four selected flights considered previously. The GLEs 5 and 69 scenarios were applied using a GLE time occurrence defined by the delay between the flight departure and the GLE. 

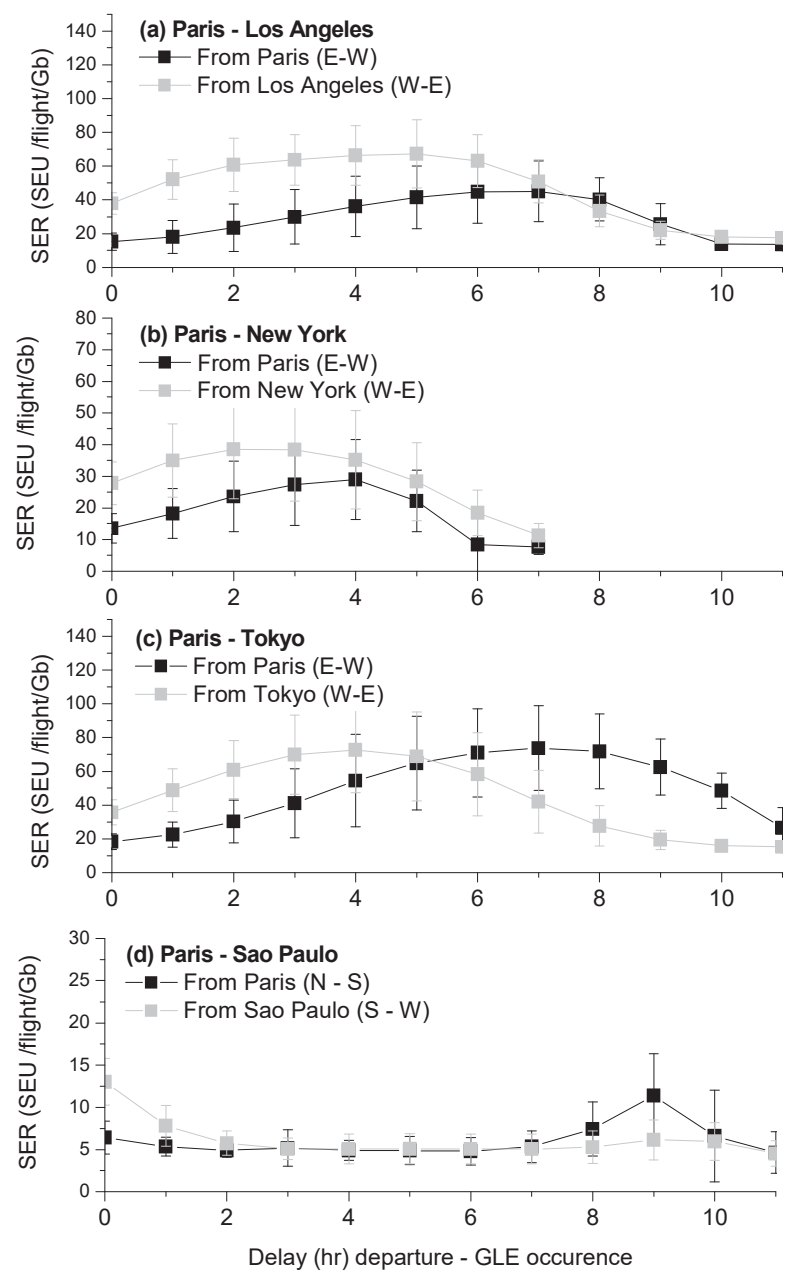

Fig. 5: Average SER calculated considering GLE 69 scenario and a GLE time occurrence, for (a) Paris - Los Angeles, (b) Paris - New York, (c) Paris - Tokyo and (d) Paris - Sao Paulo flights. The two possible flight directions are shown (from or to Paris).

Fig. 5 and Fig. 6 present average SERs for the selected flights and as a function of said delay. Uncertainties define deviations induced by the path sampling. A very large asymmetry is observed for some flight routes, the most significant examples concerning Los Angeles and Tokyo flights. Results show that North-South routes are characterized by a relatively low dissymmetry, even during a GLE. In any case, for all the considered paths, the time defining the GLE occurrence has a significant importance in the assessment of the SER. 

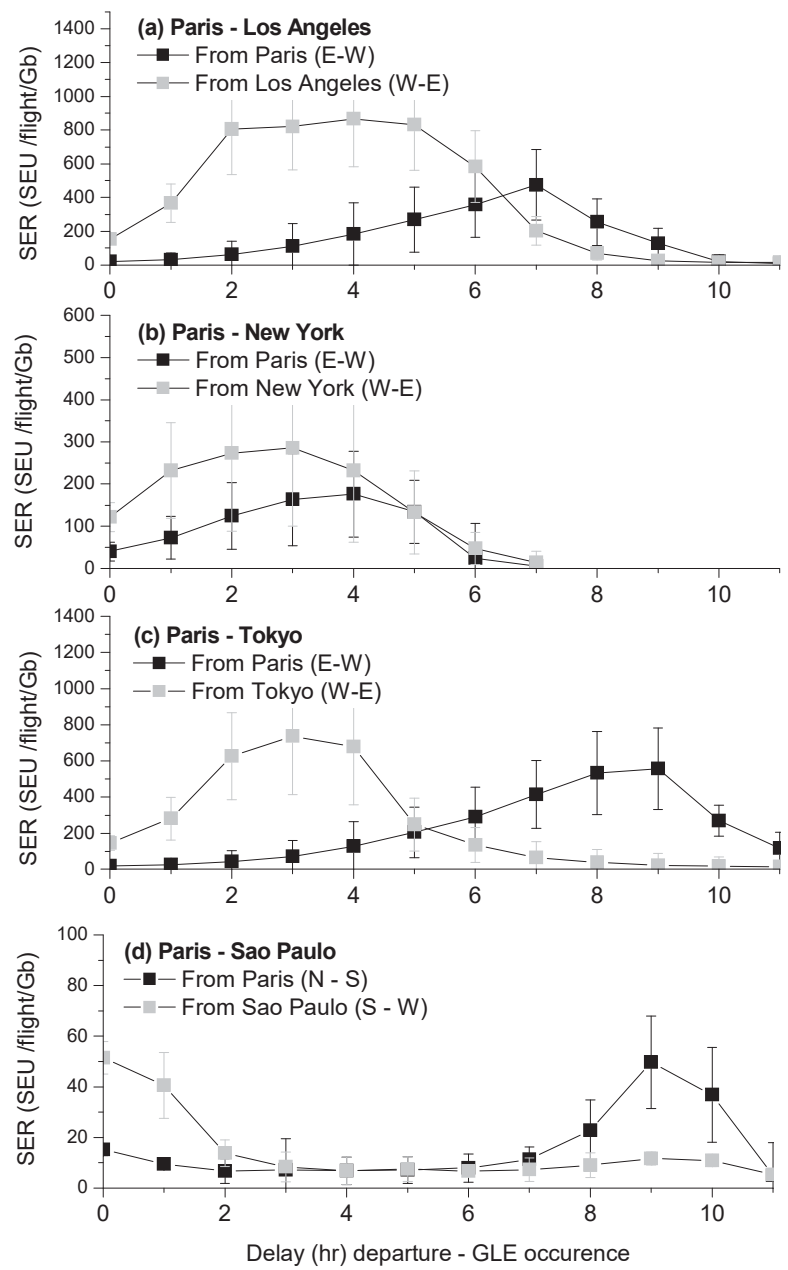

Fig.6: Average SER calculated considering GLE 5 scenario and a GLE time occurrence, for (a) Paris - Los Angeles, (b) Paris - New York, (c) Paris - Tokyo and (d) Paris - Sao Paulo flights. The two possible flight directions are shown (from or to Paris).

To extend the analyses, normalized probabilities of SER for a whole database are presented in Fig. 7. These simulations consider quiet conditions, GLE 5 and GLE 69 using a delay of 5 hours. All flights recorded in the European flight database are considered and a shift of the distribution is observed towards higher levels of SER, i.e. of the order of hundreds of SEUs/flight/Gb for the GLE 69 and of thousands for the GLE 5, while the SER is in the order of tens for quiet conditions. These results are important because they show that the SER increases significantly during a GLE event, depending to the GLE profile. 


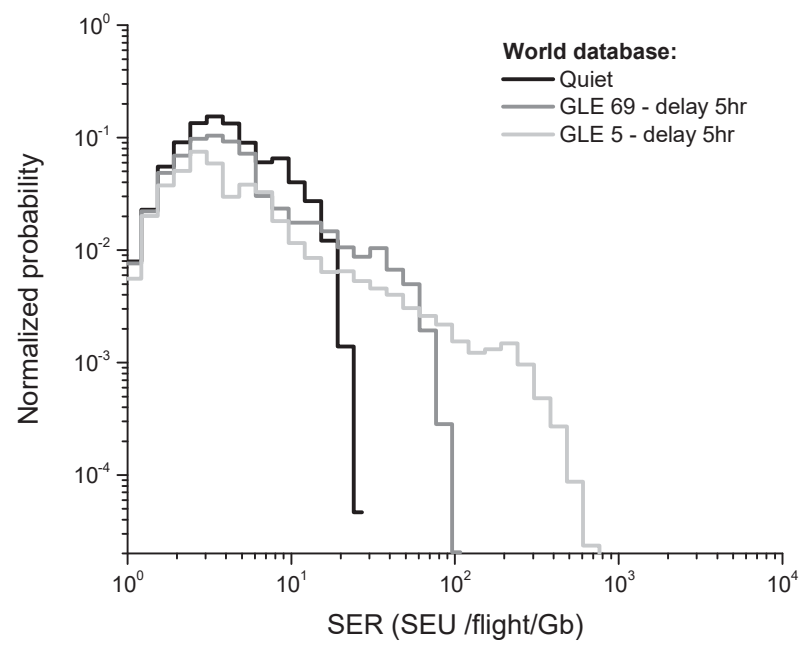

Fig. 7: SER distribution considering the quiet solar period, the GLE 5 and the GLE 69 with a delay of 5 hours, for all flights in the European flight database.

It is interesting to focus on transatlantic flights because they require fairly high latitudes. Fig. 8 presents analyses applied to Europe - North America flights, and distinguishing the flight direction (East-West and West-East). While some flights appear to be relatively unaffected by the GLE, a more detailed analysis indicates that these flights are arriving to or departing from airports in the Southern United States or from Mexico. However, the majority of flights are impacted by the GLE, by a factor of up to 5 and 30 for GLEs 69 and 5, respectively.

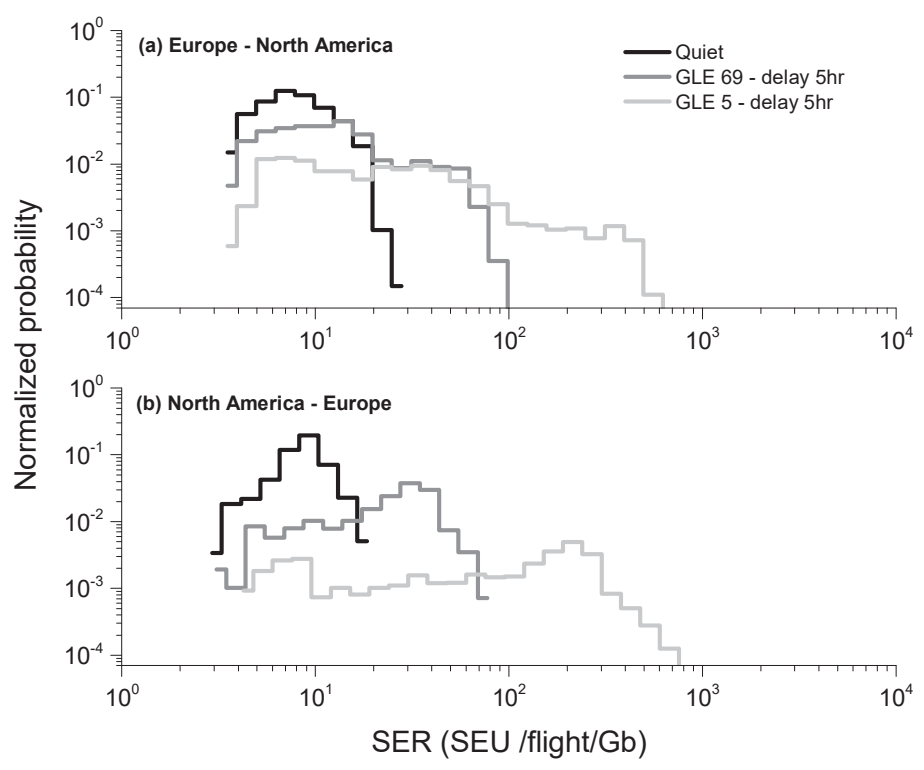

Fig. 8: SER distribution considering the quiet solar period, the GLE 5 and the GLE 69 with a delay of 5 hours. (a) Europe - North America and (b) North America - Europe flights 
Complementary calculations were performed for the South America, Asia and Africa continents. Fig. 9 presents the normalized SER probabilities considering the GLE 5 with a delay of 5 hours, and for flights Europe to/from North America, South America, Asia, Africa and Europe (internal flights). Fig. 9(a) and (b) focus on flights arriving to Europe and on flights departing from Europe, respectively. Results show that impacts of the GLE are low for South America, Africa and, especially, internal flights. In the case of Africa and South America, it is due to the mid-latitude of the paths. Indeed, flight latitudes correspond to equatorial and tropical zones implying a high geomagnetic cutoff rigidity (in the range from 8 to $12 \mathrm{GV}$ ). In the case of Asian flights, there is some similarity with the orders of magnitude observed for North America, particularly for the Siberian path. European internal flights are weakly impacted, mainly due to the low flight duration.

The West-East direction (i.e. North and South America to Europe, Europe to Asia) is systematically more impacted by the GLE. It is due to the conjugation of flight latitudes and flight durations which are longer in the West-East direction because of the atmospheric currents. There is a strong dissymmetry observed on flights in the longitude axis (i.e. Europe - Africa and, to a lesser extent, Europe - South America). Analyses show that flights flying close to or in the geographical area of Europe at the time of the GLE occurrence are further impacted. As the delay is equal to 5 hours, flights from Europe to South America and Africa are less impacted. Analyses show the main role of the SCR anisotropy function, particularly at the beginning of the event.
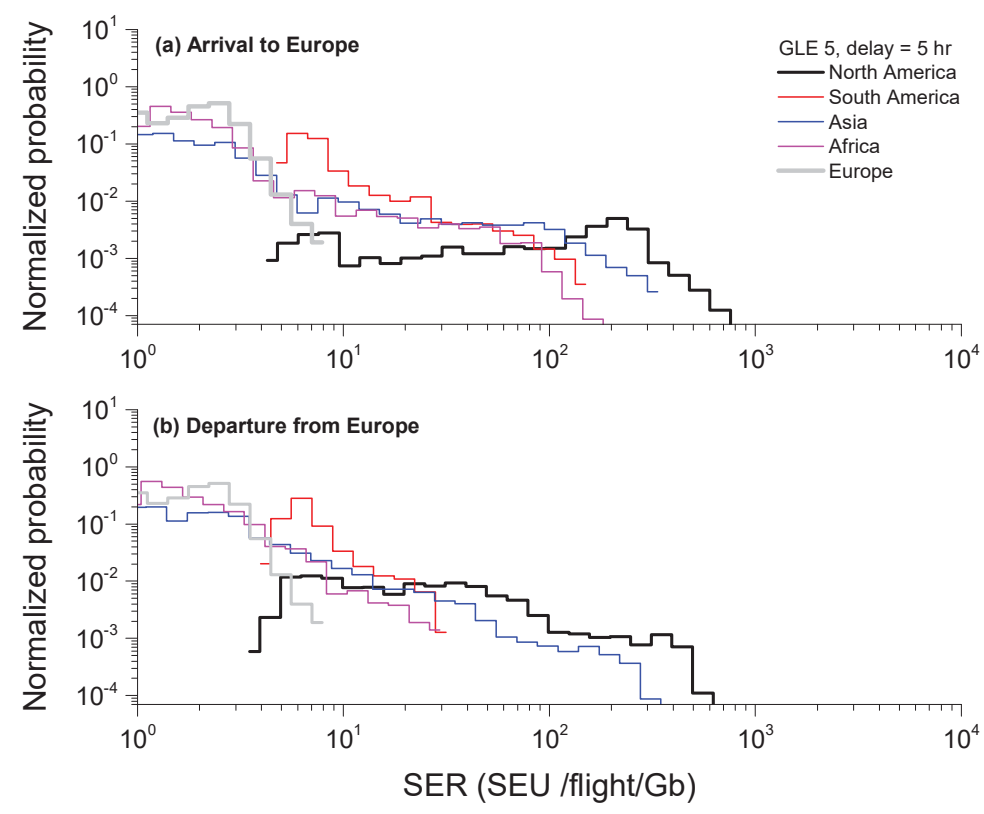

Fig. 9: Normalized SER probability considering the GLE 5 with a delay of 5 hours, and for flights Europe to/from North America, South America, Asia, Africa and Europe (internal flights); (a) Flights arriving to Europe and (b) flights departing from Europe. 


\section{Realistic simulation of the GLE impact on the air traffic}

Previous analyses had considered the GLE impact with a delay from the flight departure. In this part, GLEs were characterized by a date and a universal time, and the objective is to analyze the impact in regards to the air traffic. This approach is more adapted to assess the realistic impact, particularly in terms of impacted flight (i.e. flight for which an additional SER was observed during GLE in comparison to the value observed with quiet conditions) or normalized SER probabilities. Fig. 10 presents the dynamic SER for three selected flights and considering the GLE 69 (a) and 5 (b). The time occurrence of the GLE was considered at 12:00:00 UT. Table II describes the flights' parameters, i.e. the departure and arrival cities, the flights' durations, the distance, the maximum altitude, the time departures and the total SER in SEU number/flight/Gbit.

TABLE II: FLIGHTS' PARAMETERS, I.E. THE DEPARTURE AND ARRIVAL CITIES, FLIGHTS' DURATIONS, DISTANCE, MAXIMUM ALTITUDE, TIME DEPARTURES AND THE TOTAL SER IN SEU NUMBER PER FLIGHT PER GBIT.

\begin{tabular}{|c|c|c|c|}
\hline Flight & AFR275 & AFR454 & AFR084 \\
\hline Departure & $\begin{array}{l}\text { Tokyo } \\
\text { (RJAA) }\end{array}$ & $\begin{array}{l}\text { Sao } \\
\text { (SBGR) }\end{array}$ & $\begin{array}{l}\text { Los Angeles } \\
\text { (KLAX) }\end{array}$ \\
\hline Arrival & Paris (LFPG) & Paris (LFPG) & Paris (LFPG) \\
\hline Flight duration & $11.62 \mathrm{~h}$ & $10.42 \mathrm{~h}$ & $10.56 \mathrm{~h}$ \\
\hline Distance & $9824 \mathrm{~km}$ & $9505 \mathrm{~km}$ & $9337 \mathrm{~km}$ \\
\hline Max. altitude & $10.98 \mathrm{~km}$ & $10.42 \mathrm{~km}$ & $11.27 \mathrm{~km}$ \\
\hline Time departure & 04:18:00 UT & 22:40:00 UT & 10:38:00 UT \\
\hline $\begin{array}{l}\text { SER } \\
\text { (SEU/Gbit) }\end{array}$ & $\begin{array}{l}\text { GLE } 69: 26 \\
\text { GLE } 5: 169\end{array}$ & $\begin{array}{l}5.6 \text { (not impacted } \\
\text { by GLEs) }\end{array}$ & $\begin{array}{l}\text { GLE } 69: 19 \\
\text { GLE } 5: 126\end{array}$ \\
\hline
\end{tabular}

The flight AFR454 from Sao Paulo to Paris is not impacted by the GLE because the plane takes off after the GLE. Nevertheless, the SER rate dynamics clearly shows the latitude impact. Indeed, the flight involves a trip from the Southern to the Northern Hemisphere. At 12:00:00 (GLE occurrence), the flight AFR275 Tokyo - Paris is characterized by an altitude of 10.9 $\mathrm{km}$, a latitude of $66^{\circ} \mathrm{N}$, a longitude of $47^{\circ} \mathrm{E}$ and a cutoff rigidity of $0.63 \mathrm{GV}$. Concerning the flight AFR084 Los Angeles - Paris, the altitude is $9.75 \mathrm{~km}$, the latitude is $56.69^{\circ} \mathrm{N}$, the longitude is $4.8^{\circ} \mathrm{W}$ and the cutoff rigidity is $1.65 \mathrm{GV}$. Considering these values, particularly the cutoff rigidity (i.e. latitude/longitude) and the altitude, it is consistent that the flight Tokyo - Paris flight was highly disturbed. In the considered cases, the impact of the GLE 69 is moderate, in the order of tens of percents. The impact is more significant for the GLE 5, in the order of $1000 \%$ and $700 \%$ for the Tokyo - Paris and the Los Angeles - Paris flight, respectively. 


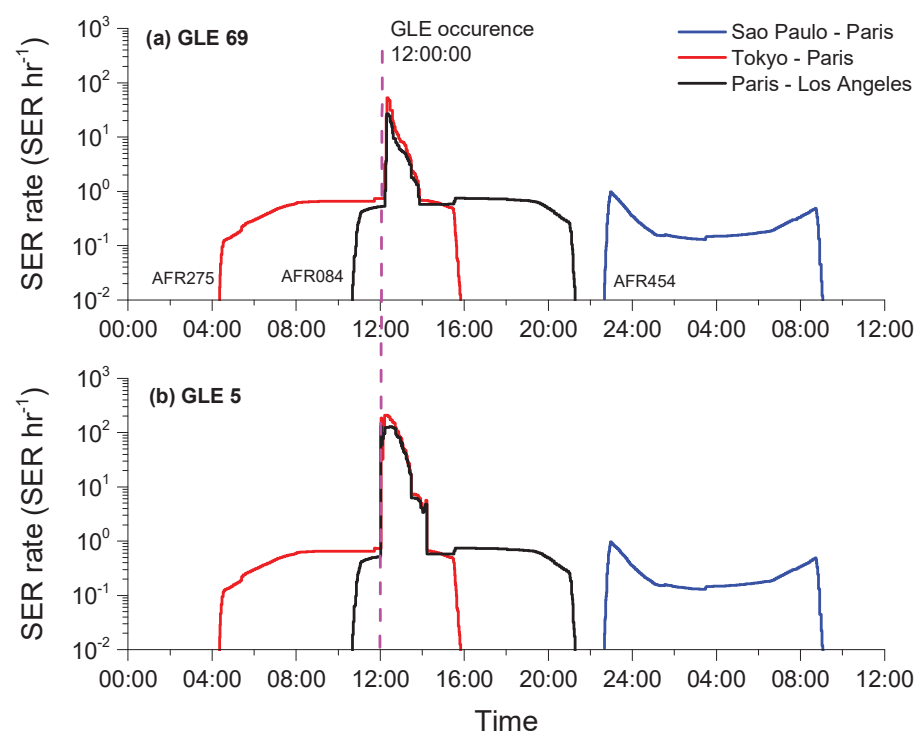

Fig. 10: Temporal variation of SER for three selected flights (AFR454 Sao Paulo - Paris, AFR275 Tokyo - Paris and AFR084 Paris - Los Angeles) and considering the GLE 69 (a) and 5 (b). The time occurrence of the GLE was considered at 12:00:00 UT.

In order to globalize the analysis, Fig. 11 presents the percentage of flights impacted during the GLE 69 (a) and 5 (b) and for several fixed universal times.

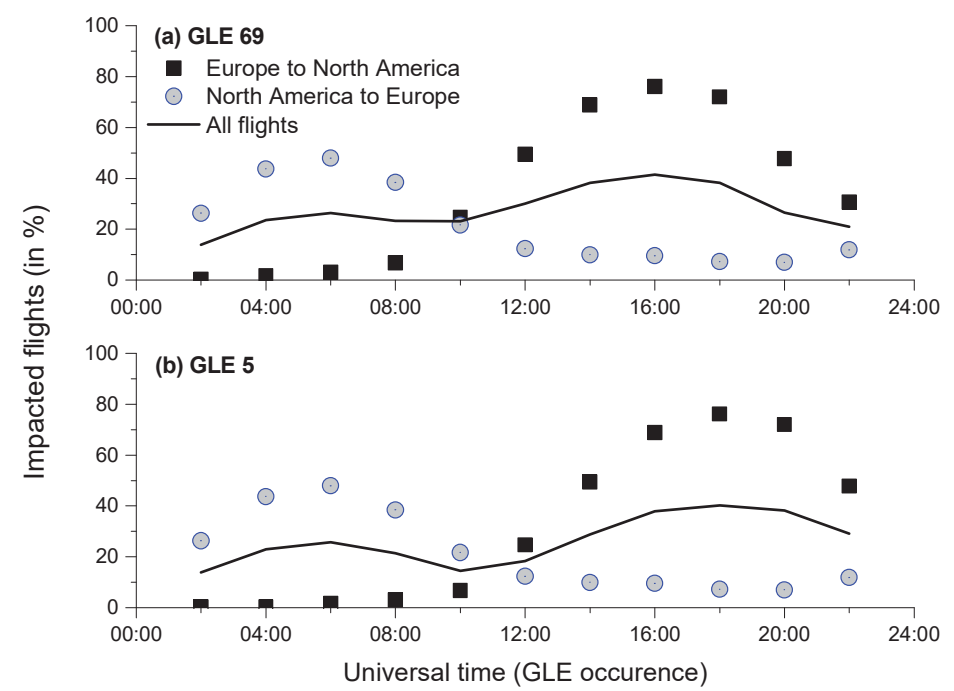

Fig. 11: Percentage of impacted flight as a function of the time GLE occurrence, (a) GLE 69 and (b) GLE 5. Results are presented for Europe - North America and North America - Europe directions.

Results were considered for Europe - North America flights and the flight direction impact was taken into account. It is 
interesting to note the influence of the time occurrence, mainly due to the traffic intensities and the direction flight. The percentage of impacted flights is high from 2:00 to 10:00 UT time for the West - East direction, whereas it is high from 12:00 to 20:00 UT time for the East - West direction. All results illustrate the complexity to assess the GLE impact because it results from the dynamic, the anisotropy and the spectrum characteristics in regards to the path details (longitude, altitude and altitude).

The percentage of flights impacted by the GLE can be significant, even more than $50 \%$. Nevertheless, an important parameter is the SER increase factor $\eta$ for a given flight, defined in (4), which corresponds to the ratio between the SER obtained with a GLE and the SER considering quiet conditions.

$\eta($ flight $)=\frac{S E R_{G L E}\left(\text { flight }, t_{\text {occurence }}\right)}{S E R_{\text {quiet }}(\text { flight })}$

Fig. 12(a) presents the percentage of impacted flights considering GLEs 5 and 69 and a GLE time occurrence fixed at 16:00 UT, as a function of the increase factor and the flight direction. It is interesting to note the important difference between both GLEs. Indeed, for the GLE 69 the value of the increase factor rises to 5-6 depending on the direction, while values can rise to 50 for the GLE 5. At 16:00 UT, the Europe - North America direction is strongly impacted, and considering that 5\% of the air traffic is impacted, the increase factor is equal to 4 and 35 for the GLE 69 and 5, respectively.

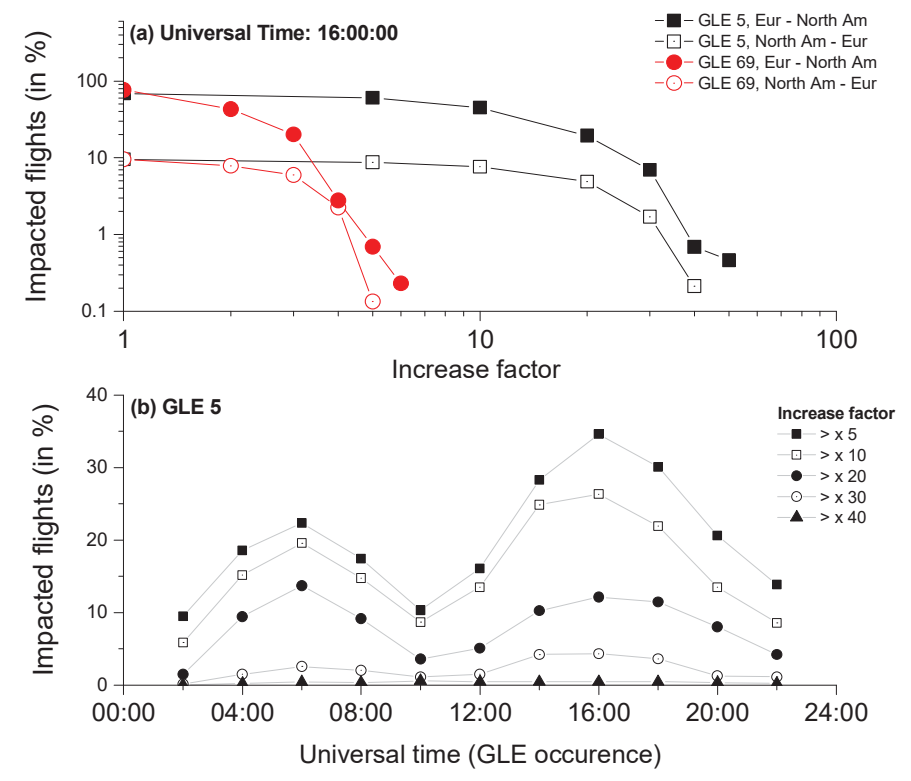

Fig. 12: (a) percentage of impacted flights considering the GLE 5 and 69 and a GLE time occurrence fixed at 16:00 UT, as a function of the increase factor and the flight direction. (b) Percentage of impacted flights as a function of the time occurrence, considering the GLE 5 and for several increase factors (5, 10, 20, 20 and 40, respectively). 
Fig. 12(b) focuses on North transatlantic flights (i.e. Europe - North America) for the GLE 5. It presents the percentage of impacted flights as a function of the time occurrence and for several increase factors $(5,10,20,20$ and 40, respectively). For instance, considering a UT occurrence of 6:00 AM and an increase factor of 20, results show that $15 \%$ of transatlantic air-traffic flights are impacted. These orders of magnitude are significant and indicate the need to consider GLE impacts on SER for realistic air traffic.

\section{DisCUSSION}

The number of flights performed globally by the airline industry steadily increase, which represents an increase of over 50 percent from a decade prior. This concerns the transatlantic international overflying traffic and specially the areas surrounding emergent countries (such as Asia). In parallel, embedded electronic systems require more and more capacities and they are based on devices that follow the technological roadmap involving increased SEE sensitivity.

Methods used to assess the SER risk focus on impact of GCRs, i.e. mainly during quiet solar period. However, some authors [42] have proposed to predict real-time atmospheric exposure from GCR and SEP events. The SEP spectrum is derived using ion flux measurements taken from the NOAA/GOES and NASA/ACE satellites.

The methodology presented in this work investigates in detail the impact of a dynamic model of extreme solar events known as GLEs. Results show that the extra SER for flights during GLEs can reach an increase factor of 100. The risk assessment is much higher than it seems, because it depends on several physical parameters, i.e. the differential SCR rigidity spectrum, the anisotropy function reflecting the distribution of SCR particles at the top of the atmosphere during the solar event, and their dynamics. The orders of magnitude of CR variations during extreme solar events raise a number of new issues, for avionics hardware but also for biological dose effects [21][22].

It is necessary to improve or to take into account the SER assessment during extreme solar events. This point highlights the importance of monitoring solar events and of developing multi-physic methods to obtain a reliable assessment of the SER.

\section{CONClusions}

This work proposes to study the GLE impact on the SER for flights. The GLE model was confronted to CR variations during GLEs 5 and 69 recorded with NMs. Firstly, measurements obtained for four NMs distributed at both sides of the North Atlantic produced coherent results with a rather significant deviation for the Ottawa station. It is complex to interpret this gap, especially considering the proximity of Ottawa with other stations as Chicago. The second comparison concerns the GLE 69, for which recorded NM data are numerous, and it allowed very sophisticated analyses of the North-South anisotropy of this event. 
The SEE calculations were based on MUSCA SEP3 calculations applied to bulk $90 \mathrm{~nm}$ COTS SRAMs. Analyses focus on three flights for which calculations can be compared with experimental data obtained during quiet solar conditions.

Based on GLE and SEU occurrence validated models, investigations were performed to assess the SER during a GLE. A first approach consists in considering four selected flights characterized by a high flight number representative to the world-air traffic (Paris - Los Angeles, New York, Sao Paulo and Tokyo), and for the GLEs 5 and 69. Considering the GLE impact, the main conclusion is that SER assessment needs to consider the route profile and the solar event characteristics.

As detailed, GLEs vary in energy spectrum but it is possible to consider that, approximately once per year, particles are sufficiently energetic to significantly increase the flux of secondary cosmic rays on the ground and at aircraft cruising altitudes. The main conclusion is that the GLE impact must be considered in the SEE risk assessment, which implies a physical description of the additional SCR and a realistic consideration of the air traffic.

\section{REFERENCES}

[1] Grieder v, Cosmic Rays at Earth: Researcher's Reference Manual and Data Book, 1st ed., pp. 1093, Elsevier, 2001.

[2] Reitz G., Radiation environment in the stratosphere. Radiat. Prot. Dosimetry; vol. 48, no. 1, pp. 5 - $20,1993$.

[3] Hubert G., D. Régis, A. Cheminet, M. Gatti and V. Lacoste, Multi-physics modelling contributions to investigate the atmospheric cosmic rays impact on the single event upset sensitivity along the scaling trend of CMOS technologies, Radiat. Prot. Dosimetry, vol. 161 (1-4), pp. 290 - $294,2014$.

[4] Olazábal A. J. and J.P. Guerra, Multiple Cell Upsets Inside Aircrafts. New Fault-Tolerant Architecture, IEEE Trans. Aerosp. Electron. Syst., vol. 55, no. 1, pp. $32-342,2019$.

[5] Ghodbane A., M. Saad, C. Hobeika, J.F. Boland and C. Thibeault, Design of a tolerant flight control system in response to multiple actuator control signal faults induced by cosmic rays, IEEE Trans. Aerosp. Electron. Syst., vol. 52, no. 2, pp. 681 - 697, 2016.

[6] Zhang H., W. S. Gray, O. R. González and A.V. Lakdawala, "Tracking Performance of a Recoverable Flight Control System in Neutron Environments", IEEE Trans. Aerosp. Electron. Syst., vol. 45, no. 1, pp. $321-335,2009$.

[7] Ghelfi, et al., Neutron monitors and muon detectors for solar modulation studies: 2. $\phi$ time series. Advances in Space Research, 60(4), 833-847, 2017.

[8] Maurin, D., et al., Neutron Monitors and Muon counters for Solar modulation Studies: Assessment of critical parameters in count rate calculations. Advances in Space Research, 60(4), 833-847, 2017.

[9] Gleeson L. and W. Axford, Solar Modulation of Galactic Cosmic Rays, Astrophysical Journal, vol. 154, pp. 1011 - $1026,1968$.

[10] Van Allen J. A. and J. R. Winckler, Spectrum of low-rigidity cosmic rays during the solar flare of February 23 1956, Phys. Rev., vol. 106, no. 5, pp. 1072 $-1073,1957$.

[11] Smart D. F. and M. A. Shea, Probable pitch angle distribution and spectra of the 23 February 1956 solar cosmic ray event, Proc. 21 st Int. Cosmic Ray Conf., vol. 5, pp. $257-260,1990$.

[12] Normand E., Correlation of In-flight Neutron Dosimeter and SEU Measurements with Atmospheric Neutron Model, IEEE Trans. Nucl. Sci., vol. 48, no. 6, pp. $1996-2003,2001$.

[13] Normand, E., J. L. Wert, D. L. Oberg, P. R. Majewski, P. Voss, and S. A. Wender, Neutron-Induced Single Event Burnout in High Voltage Electronics, IEEE Trans. Nucl. Sci, vol. 44, no. 6, pp. $2358-2366,1997$.

[14] Olsen, J., P. E. Becher, P. B. Fynbo, P. Raaby, and J. Schultz, Neutron-Induced Single Event Upsets in Static RAMs Observed at 10 km Flight Altitude, IEEE Trans. Nucl. Sci., vol. 40, no. 2, pp. $74-77,1993$.

[15] Process management for avionics - Atmospheric radiation effects - Part 2: Guidelines for single event effects testing for avionics systems, International Standard, IEC 62396-2, 2017.

[16] Process management for avionics - Management plan - Part 1: Preparation and maintenance of an electronic components management plan, International Standard, IEC /TS 62239-1, 2018.

[17] Process management for avionics - Atmospheric radiation effects - Part 1: Accommodation of atmospheric radiation effects via single event effects within avionics electronic equipment, International Standard, IEC 62396-1, 2016.

[18] Usoskin I. G. and G. A. Kovaltsov, Occurrence of Extreme Solar Particle event: assessment from historical proxy data, Astrophys. J., vol. 757, no. 92, 2012.

[19] Plainaki C., A. Belov, E. Eroshenko, H. Mavromichalaki and V. Yanke, Modeling ground level enhancements: Event of 20 January 2005 , J. Geophys. Res., vol. 112, A04102, 2007.

[20] Hubert G. and A. Cheminet, Radiation Effects Investigations Based on Atmospheric Radiation Model (ATMORAD) Considering GEANT4 Simulations of Extensive Air Showers and Solar Modulation Potential, Radiat. Res., vol. 184, no. 1, pp. 83 - 94, 2015.

[21] Hubert G. and S. Aubry, Atmospheric Cosmic-Ray Variation and Ambient Dose Equivalent Assessments Considering Ground Level Enhancement Thanks to Coupled Anisotropic Solar Cosmic Ray and Extensive Air Shower Modeling, Radiat. Res., vol. 188, no. 5, pp. 517 - 531, 2017.

[22] Hubert G. and S. Aubry, Analysis of Exposure to Solar and Galactic Cosmic Radiations of Flights Representative of the European International Air Traffic, Radiat. Res., vol. 190, no. 3, pp. $271-281,2018$.

[23] Hubert G. and S. Aubry, Analysis of the Forbush Decreases and Ground-Level Enhancement on September 2017 Using Neutron Spectrometers Operated in Antarctic and Midlatitude Stations, Journal of Geophysical Research, JGR Space Physics, vol. 124, no. 1, pp. 661 - 673, 2019.

[24] Miayasaka H., E. Takahashi, S. Shimoda, Y. Yamada, I. Kondo et al., The solar Event of 20 January 2005 observed with the Tibet YBJ Neutron Monitor Observatory, Proc. 29th Int. Cosmic Ray Conf. Pune, vol. 1, pp. 241 - 244, 2005.

[25] Usoskin I., A. Tylka, G. Kovaltsov and W. Dietrich, Ionization effect of strong solar particle events: Low-middle atmosphere, Proc. 31 st ICRC, 2009. 
[26] Meyer P., E. Parker and J. Simpson, Solar cosmic rays of February 1956 and their propagation through interplanetary space, Phys. Rev., vol. 104, no. 3, pp. $768-783,1956$.

[27] Lantos P., Radiation doses potentially received on-board aeroplanes during recent solar particle events, Radiat. Prot. Dosimetry, vol. 118, no. 4, pp. 363 $374,2006$.

[28] Lantos P. and N. Fuller, Semi-empirical model to calculate potential radiation exposure on board airplane during solar particle events, IEEE Trans. On Plasma Sci., vol. 32, no. 4, pp. 1468 - 1477, 2004.

[29] Hubert G., S. Duzellier, C. Inguimbert, C. Boatella-Polo, F. Bezerra, and R. Ecoffet, Operational SER calculations on the SAC-C orbit using the Multi SCAles Single Event Phenomena Predictive Platform (MUSCA SEP3), IEEE Trans. Nucl. Sci., vol. 56, no.6, pp. 3032 - $3042,2009$.

[30] Hubert G., R. Velazco, C. Frederico, A. Cheminet, C. Silva-Cardenas, L. V. E. Caldas, F. Pancher, V. Lacoste, F. Palumbo, W. Mansour, L. Artola, F. Pineda and S. Duzellier, Continuous high-altitude measurements of cosmic ray neutron and SEU/MCU at various locations: correlation and analyses based-on MUSCA-SEP3, IEEE Trans. Nucl. Sci., vol. 60, no. 4, pp. 2418 - 2426, 2013.

[31] SRIM, http://srim.org/

[32] Agostinelli S., et al. GEANT4-a simulation toolkit, Nucl. Instrum. Methods Phys. Res. A, vol. 506, no. 3, pp. 250 - $303,2003$.

[33] Allison J., et al. Geant4 developments and applications, IEEE Trans. Nucl. Sci., vol. 53, no. 1, pp. $270-278,2006$.

[34] Peronnard P., R. Velazco and G. Hubert, Real-life SEU experiments on 90nm SRAMs in Atmospheric Environment: measure vs. predictions done by means of MUSCA SEP3 platform, IEEE Trans. Nucl. Sci., vol. 56, no. 6, pp. $3450-3455,2009$.

[35] Hubert G., R. Velazco and P. Peronnard, A generic platform for remote accelerated tests and high altitude SEU experiments on advanced ICs: Correlation with MUSCA SEP3 calculations, 15th IEEE Int. On-Line Testing Symp., pp. 180, 2009.

[36] Hands A., P. Morris, C. Dyer, K. Ryden and P. Truscot, Single event effects in poxer MOSFETs ad SRAMs dur to 3 MeV, 14 MeV and fissions neutrons, IEEE Trans. Nucl. Sci., vol. 58, no. 3, pp. $952-958,2011$.

[37] Artola L., R. Velazco, G. Hubert, W. Mansour, F. Pancher, S. Duzellier, T. Nuns, F. Bezerra and B. Guerard, In Flight SEU/MBU Sensitivity of Commercial Nanometric SRAMs: Operational Estimations, IEEE Trans. Nucl. Sci., vol. 58, no. 6, pp. $2644-2651,2011$.

[38] Clemente J. A., F. J. Franco, F. Villa, M. Baylac, S. Rey, H. Mecha, J. A. Agapito, H. Puchner, G. Hubert and R. Velazco, Statistical Anomalies of Bitflips in SRAMs to Discriminate SBUs from MCUs, IEEE Trans. Nucl. Sci., vol. 63, no. 4, pp. 2087 - 2094, 2016.

[39] Clemente J.A., G. Hubert, F. J. Franco, F. Villa, M., Baylac, H. Mecha, H. Puchner and R. Velazco, Sensitivity Characterization of a COTS 90-nm SRAM at Ultralow Bias Voltage, IEEE Trans. Nucl. Sci., vol. 64, no. 8, pp. $2188-2195,2017$.

[40] Clemente J.A., G. Hubert, J. Fraire, F. J. Franco, F. Villa, S. Rey, M. Baylac, H. Puchner, H. Mecha, R. Velazco, SEU Characterization of Three Successive Generations of COTS SRAMs at Ultralow Bias Voltage to 14.2-MeV Neutrons, IEEE Trans. Nucl. Sci., vol. 65, no. 8, pp. 1858 - 1865, 2018.

[41] www.eurocontrol.int

[42] Mertens C. J., W. K. Tobiska, D. Bouwer, B. T. Kress, M. Wiltberger, S. C. Solomon, and J. J. Murray, Development of the Nowcast of Atmospheric Ionizing Radiation for Aviation Safety (NAIRAS) model, 1st AIAA Atmospheric and Space Environments Conf., 2009.

\section{BIOGRAPHIES}

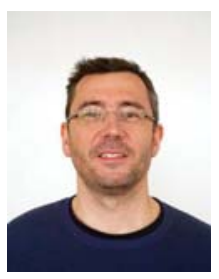

Guillaume HUBERT received his M. S. Degree in Theoretical Physics from the Pierre et Marie Curie University (Paris VI), his Ph.D. degree from the Montpellier University in 2002 and his capacitance to supervise PhD from the Toulouse University in 2010. From 2002 to 2007, he was a research physicist at European Aeronautic Defense and System (EADS) group. Since 2007, he joined ONERA (the French Aerospace Lab.), at the Physics, Instrumentation, environment and Space Department (DPHY). Its research activities include 1) Single Event Effect modelling based on multi-physics and 2) atmospheric radiation environment studies based on world-network operating Cosmic Ray spectrometers in high-altitude and nuclear transport simulations applied to atmospheric air-showers. He is author and co-author more than 120 international journal publications and conference proceeding in the fields of SEE and Cosmic Rays. In terms of research projects, he participates or has participated in International, European or national projects. He is currently principal investigator of the CHINSTRAP polar project in which he has conducted three summer campaigns in Antarctica.

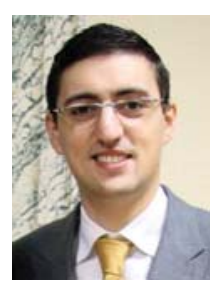

Juan Antonio Clemente received his computer science degree from Universidad Complutense de Madrid (UCM), Madrid, Spain, in 2007; and his Ph.D. in 2011. He is Assistant Professor in the Computer Architecture Department-UCM and Researcher with the GHADIR research Group. His research is focused on the study of Single Event Effects (SEE) tolerance of digital circuits, especially COTS memories, and their use in harsh environments such as space. For conducting this research, he collaborates with the TIMA Laboratory, in the Grenoble-Alpes university, Grenoble, France; and with the ONERA (the French Aerospace Lab), in Toulouse, France.

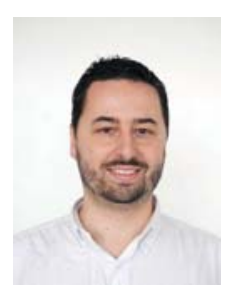

Sébastien Aubry is engineer from ENAC (the French National Civil Aviation University) with 20 years' experience in Air Traffic management studies, including modelling, simulation and evaluation. He is member of various projects regarding aeronautics, notably in the European (SESAR, Clean Sky, H2020) and French (DGAC with Thales, Airbus) context. Since 2019, he is Air Transport System and Avionics Program Director at ONERA. 
Fig. 1: Cosmic ray variations measured and calculated during the GLE 69 in the South Pole (a), McMurdo (b), Thule (c) and Tibet stations (d).

Fig. 2: Cosmic ray variations measured and calculated during the GLE 5 in Leeds (a), Stockholm (b), Chicago (c) and Ottawa (d).

Fig. 3: Distribution of the SEU number calculated for the Los Angeles - Paris, Buenos Aires - Paris and Madrid - Buenos Aires flights. The measured SEU numbers recorded during flights were added.

Fig. 4: Normalized probability of SER for (a) Paris- Los Angeles, (b) Paris- New York, (c) Paris - Tokyo and (d) Paris - Sao Paulo routes. Results are presented in distinguishing the flight direction (from / to Paris). Average, minimum and maximum SER were added.

Fig. 5: Average SER calculated considering GLE 69 scenario and a GLE time occurrence, for (a) Paris - Los Angeles, (b) Paris - New York, (c) Paris - Tokyo and (d) Paris - Sao Paulo flights. The two possible flight directions are shown (from or to Paris).

Fig.6: Average SER calculated considering GLE 5 scenario and a GLE time occurrence, for (a) Paris - Los Angeles, (b) Paris - New York, (c) Paris - Tokyo and (d) Paris - Sao Paulo flights. The two possible flight directions are shown (from or to Paris).

Fig. 7: SER distribution considering the quiet solar period, the GLE 5 and the GLE 69 with a delay of 5 hours, for all flights in the European flight database.

Fig. 8: SER distribution considering the quiet solar period, the GLE 5 and the GLE 69 with a delay of 5 hours. (a) Europe - North America and (b) North America - Europe flights

Fig. 9: Normalized SER probability considering the GLE 5 with a delay of 5 hours, and for flights Europe to/from North America, South America, Asia, Africa and Europe (internal flights); (a) Flights arriving to Europe and (b) flights departing from Europe.

Fig. 10: Temporal variation of SER for three selected flights (AFR454 Sao Paulo - Paris, AFR275 Tokyo - Paris and AFR084 Paris - Los Angeles) and considering the GLE 69 (a) and 5 (b). The time occurrence of the GLE was considered at 12:00:00 UT.

Fig. 11: Percentage of impacted flight as a function of the time GLE occurrence, (a) GLE 69 and (b) GLE 5. Results are presented for Europe - North America and North America - Europe directions.

Fig. 12: (a) percentage of impacted flights considering the GLE 5 and 69 and a GLE time occurrence fixed at 16:00 UT, as a function of the increase factor and the flight direction. (b) Percentage of impacted flights as a function of the time occurrence, considering the GLE 5 and for several increase factors (5, 10, 20, 20 and 40, respectively). 